\title{
Editor’s Foreword
}

$\mathrm{D}$ EAR readers and authors, welcome to the inaugural issue of Journal on Selected Topics in Nano Electronics and Computing (JSTNEC). I would like to start this editorial introduction with some rather self-evident statement. No one has any doubt in the fact that the computer and IT revolution is based on an exponential growth of technological progress. As is well known, the performance of semiconductor devices doubles every nearly eighteen months, and after that the speed and memory size of computers and mobile gadgets, wireless communication capacity, internet traffic and other quality indexes of electronic devices underlying the IT progress double every few months or years too.

This technological progress requires more and more sophisticated and highly efficient electronic chips, and their design and further development, in turn, rely upon modern achievements in the area of information and computer technology. That is why this journal, the JSTNEC, has been decided to be launched with the focus on nano-electronics together with its close relation to the computing field. With the emergence of novel computing devices, such a periodical is expected to receive high demand. In other words, despite a great many existing journals, both regular and electronic online, open access included, in the areas of nano-electronics and nano-technology, on the one part, and in computing and IT, on the other part, it is hoped that this newly launched Journal would be of interest for a wide readership audience.

The Journal's scope is supposed to include the following selected topics: solid-state electronics (including semiconducting electronics, superconducting electronics, spintronics, optoelectronics, and oxide electronics); physical phenomena in solid-state micro- and nanostructures, metal, semiconductor and dielectric thin films and coatings; physical basis of plasma technology, including modification of the surface properties and deposition of thin films; related issues of nanotechnology, including (yet not limited to), for instance, studies of advanced materials based on nanocomposites using natural compounds; automated systems of scientific research, data processing and control; ubiquities computing, Smart Spaces and Internet of Things; big data, knowledge management and ontology engineering; self-organizing resource restricted networks and sensor networks, including wireless sensor networks; mathematical modeling, numerical methods and software, both in the field of nano-electronics, and in computing.

The above topics are divided into two journal sections, namely, Nanoelectronics and Related Issues of Nanotechnology and Computing and Related Issues of
Information and Communication Technology. And the last but not the least is a special section named Teaching Strategy and Computing Curricula which is destined for articles, communications and reviews devoted to various aspects of IT and nano-electronics in the sphere of higher education.

The editorial board of JSTNEC is intended to provide a forum for exchange of information on all the multifaceted aspects of the above topics in various formats, including full length research papers, brief communications and survey papers. The editorial policy will be aimed to support both mature and new cutting edge research provided that they have a strong experimental or theoretical basis. Although JSTNEC is published two times a year, in order to ensure rapid dissemination of information, we aim at completing the review process of each paper as soon as possible, at least within three months of initial submission. I draw your attention to the fact that, as this is an inaugural journal issue, the articles presented herein were not peerreviewed, and they were solicited from a selected group of researchers in the fields of IT, computing and electronics.

Finally, we wish to encourage contributions from the scientific community to ensure a continued success of the journal. Authors and reviewers are always welcome. We will also always welcome suggestions that could improve the quality of the journal and make it even more attractive for a wide range of readers and experts in the fields of IT, computing and nanoelectronics

Thank you.
Genrikh Stefanovich

Editor-in-Chief, Professor, Doctor of Physics and Mathematics

December 2013 